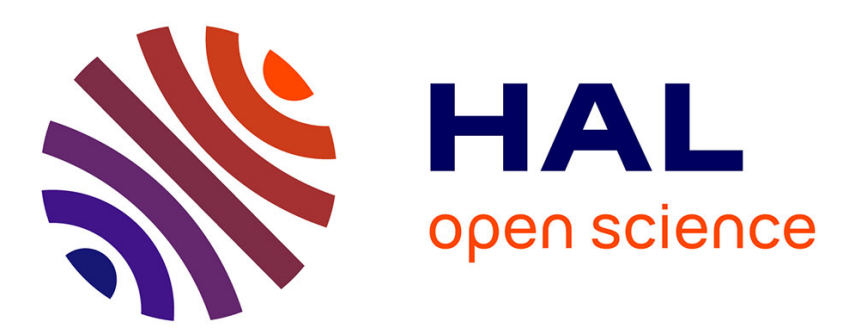

\title{
On imprecision in relation to uncertainty importance measures
}

\author{
R. Flage, Terje Aven, Piero Baraldi, Enrico Zio
}

\section{To cite this version:}

R. Flage, Terje Aven, Piero Baraldi, Enrico Zio. On imprecision in relation to uncertainty importance measures. ESREL 2011, Sep 2011, Troyes, France. pp.2250-2255. hal-00658103

\section{HAL Id: hal-00658103 \\ https://hal-centralesupelec.archives-ouvertes.fr/hal-00658103}

Submitted on 12 Jan 2012

HAL is a multi-disciplinary open access archive for the deposit and dissemination of scientific research documents, whether they are published or not. The documents may come from teaching and research institutions in France or abroad, or from public or private research centers.
L'archive ouverte pluridisciplinaire HAL, est destinée au dépôt et à la diffusion de documents scientifiques de niveau recherche, publiés ou non, émanant des établissements d'enseignement et de recherche français ou étrangers, des laboratoires publics ou privés. 


\title{
On imprecision in relation to uncertainty importance measures
}

\author{
R. Flage \& T. Aven \\ University of Stavanger, Norway \\ P. Baraldi \\ Polytechnic of Milan, Italy \\ E. Zio \\ Ecole Centrale Paris and Supelec, France \\ Polytechnic of Milan, Italy
}

\begin{abstract}
A number of uncertainty importance measures have been proposed in the literature to extend classical risk and reliability importance measures in the presence of epistemic uncertainty. Uncertainty importance measures typically reflect to what degree uncertainty about risk and reliability parameters at the component level influences uncertainty about parameters at the system level. The definition of these measures is typically founded on a Bayesian perspective where subjective probabilities are used to express epistemic uncertainty; hence, they do not reflect the effect of imprecision in probability assignments, as captured by alternative uncertainty representation frameworks such as imprecise probability, possibility theory and evidence theory. In the present paper we consider the issue of imprecision in relation to uncertainty importance measures. We define an imprecision importance measure to evaluate the effect of removing imprecision - in the present paper focusing on imprecision removal to the extent that no epistemic uncertainty remains; as further work we suggest to also consider the more general case of imprecision removal to the extent that a probabilistic representation of uncertainty remains. A numerical example is presented to illustrate the suggested measure in the case of a possibilistic uncertainty representation.
\end{abstract}

\section{INTRODUCTION}

The use of importance measures (IM) is an integral part of reliability and risk analysis. IM are used to study the effect on system level reliability or risk parameters of altering component level parameters. A number of uncertainty importance measures (UIM) have also been proposed in the literature. These extend the 'classical' reliability and risk IM in the presence of epistemic uncertainty. UIM are used to study to what degree uncertainty about risk and reliability parameters at the component level influences uncertainty about parameters at the system level.

Consider the following setting: We are interested in the quantity $Y$, possibly a vector, and introduce a model $g(X)$ which links $n$ input quantities $X=\left(X_{1}, X_{2}, \ldots, X_{n}\right)$ to $Y$. In the present paper, particular attention is paid to the case $p=g(q)$, where $p$ and $q$ are reliability or risk parameters at the system and component level, respectively. Typically $p$ and $q$ have the interpretation of long-run frequencies, e.g. the fraction of time a system and its components are functioning, respectively. This interpretation is seen, for example, in the probability of frequency approach to risk analysis (Kaplan \& Garrick, 1981).

Classical IM are used to analyze changes to $p$ given changes to $q$. For example, the so-called 'improvement potential' of component $i$ is defined as the change to the system availability $p$ when the component availability $q_{i}$ is set equal to 1 . Furthermore, the Birnbaum IM is defined as the partial derivative of $\mathrm{p}$ with respect to $q_{i}$.

UIM are typically founded on a Bayesian perspective. A subjective probability distribution $F$ is introduced for $q$ and propagated through a model $g$. The result is a probability distribution of $p$, and UIMs are used to analyse changes to the distribution of $p$ given changes to $F$. Reference is made to Section 2 for a brief review of IM and UIM.

In a Bayesian perspective subjective probabilities express epistemic uncertainty; hence, they do not reflect imprecision in probability assignments. The term imprecision here labels the phenomenon captured by a wide range of extensions of the classical theory of probability, including lower and upper previsions (Walley, 1991), belief and plausibility functions (Dempster, 1967; Shafer, 1976), possibility measures (Dubois \& Prade, 1988), robust Bayesian methods (Berger, 1984), p-boxes (Ferson et al., 2003) and interval probability (Weichselberger, 2000).

One much studied type of UIM is that reflecting the effect on system level parameter uncertainty of removing component level parameter uncertainty. For example, for a probability distribution $F$ of component level parameters $q$ which propagated through a model $g$ induces a probability distribution of the 
system level parameter $p$, this type of UIM evaluates changes to the distribution of $p$ by assuming $q_{i}$ known for some $i$. Of course, the value of $\mathrm{q}_{\mathrm{i}}$ cannot be specified with certainty and so the resulting measure becomes a function of $q_{i}$. An example is the measure $\operatorname{Var}(p)-\operatorname{Var}\left(p \mid q_{i}\right)$, expressing the reduction in the variance of the system level parameter $p$ that is achieved by specifying the value of the component level parameter $q_{i}$. One way to proceed is to consider the expected value of the above measure, as done by Iman (1987), namely:

$\operatorname{Var}(p)-E\left[\operatorname{Var}\left(p \mid q_{i}\right)\right]=\operatorname{Var}\left(E\left[p \mid q_{i}\right]\right)$.

Aven \& Nøkland (2010) investigate the link between UIM and traditional IM. In doing so they distinguish between the cases that $X$ and $Y$ as introduced above are (a) observable events and quantities, such as the occurrence of a system failure and the number of system failures, and (b) unobservable parameters, such as $p$ and $q$. Based on their findings a combined set of IM and UIM is defined.

In the present paper we consider the case that a distribution pair $H_{q}$ is introduced for $q$. We may for example have $H_{q}=\left[N_{q}, \Pi_{q}\right]$, where $N_{q}$ and $\Pi_{q}$ are the cumulative necessity and possibility distributions of $q$, respectively; or $H_{q}=\left[\mathrm{Bel}_{q}, \mathrm{Pl}_{q}\right]$, where $\mathrm{Bel}_{q}$ and $\mathrm{Pl}_{q}$ are the cumulative belief and plausibility distributions of $q$, respectively; or $H_{q}=\left[H_{q}^{l}, H_{q}{ }^{u}\right]$ where $H_{q}{ }^{1}$ and $H_{q}{ }^{u}$ are lower and upper imprecise probability distributions of $q$, respectively. Defining the imprecision of a distribution pair as the area between its lower and upper cumulative distributions, we define an imprecision importance measure (IIM) that evaluates the effect on system level parameter imprecision of removing component level parameter imprecision. Two extents of imprecision removal are possible:

i. Removal of imprecision to the extent that a probabilistic representation remains.

ii. Removal of imprecision to the extent that no epistemic uncertainty remains.

The latter case may be seen as a special case of the former. The definition of an IIM in terms of imprecision removal is associated with an analogous problem as was seen above for uncertainty removal in the case of UIM; namely, the measure can be defined but neither the specific value of a component level parameter nor its probability distribution can really be specified. We are led to consider, respectively:

I. The least and most specific probability distributions consistent with $H_{q}$.

II. The IIM as a function of $q_{i}$.
In the following we refer to these as type I and type II measures. In the present paper we focus on the type II measure.

The remainder of the paper is organized as follows: In Section 2 we review some basic classical IM and some UIM. In Section 3 we review the concepts of uncertainty and imprecision, as well as their representation. In Section 4 we define an IIM as indicated above, and in Section 5 the suggested measure is evaluated in terms of a numerical example. Section 6 provides a discussion and some directions for further work.

\section{CLASSICAL IM AND UIM}

There are essentially two fundamental classical IM: the 'improvement potential' of a component, describing the effect on the system reliability of making the component perfectly reliable; and the Birnbaum IM, reflecting the effect on system reliability of an incremental change in the reliability of a component. The improvement potential of a component is defined by (e.g. Aven \& Jensen, 1999; Rausand \& Høyland, 2004)

$h\left(1_{i}, q\right)-h(q)$,

where $h(q)$ is the system reliability function, i.e. an expression of $p$ as a function of $q$; and $h\left(1_{i}, q\right)=h\left(q_{1}, \ldots, 1_{i}, \ldots q_{n}\right)$, i.e. the system reliability function when component $i$ is perfectly reliable. The IMs referred to as risk achievement worth (RAW) and risk reduction worth (RRW) (e.g. Cheok et al., 1998; Rausand \& Høyland, 2004; Zio, 2009) represent minor adjustments of the improvement potential IM. The Birnbaum IM is defined by (e.g. Aven \& Jensen, 1999; Rausand \& Høyland, 2004; Zio, 2009)

$\frac{\partial h(q)}{\partial q_{i}}$,

i.e. as the partial derivative of the system reliability with respect to $q_{i}$. The improvement potential IM is most relevant in the design phase of a system, whereas the Birnbaum IM is most relevant in the operational phase (Nøkland \& Aven, unpubl.). See Rausand \& Høyland (2004) and Zio (2009) for a more in-depth review of classical IMs.

UIMs were described to some extent in Section 1. The UIM by Iman (1987) is variance-based and hence an example of a measure in one of the three categories described by Borgonovo (2006):

i. Nonparametric techniques (input-output correlation)

ii. Variance-based importance measures

iii. Moment-independent sensitivity indicators.

See Borgonovo (2006) for a more in-depth review of UIMs. 


\section{UNCERTAINTY, IMPRECISION AND ITS REPRESENTATION}

In engineering risk analysis a distinction is commonly made between aleatory (stochastic) and epistemic (knowledge-related) uncertainty (e.g. Apostolakis, 1990; Helton \& Burmaster, 1996). Aleatory uncertainty refers to variation in populations. Epistemic uncertainty refers to lack of knowledge about phenomena and usually translates into uncertainty about the parameters of a model used to describe random variation. Whereas epistemic uncertainty can be reduced, aleatory uncertainty cannot and for this reason it is sometimes called irreducible uncertainty (Helton \& Burmaster, 1996).

Traditionally, limiting relative frequency probabilities are used to describe aleatory uncertainty and subjective probabilities are used to describe epistemic uncertainty. However, as described in Section 1 , several alternatives to probability as representation of epistemic uncertainty have been suggested, the motivation being to capture imprecision in subjective probability assignments. Imprecision here refers to inability to precisely specify a probability (distribution).

Numerical possibility distributions can encode special convex families of probability measures (Dubois, 2006). In possibility theory, uncertainty and imprecision is represented by a possibility function $\pi$. For each element $\omega$ in a set $\Omega, \pi(\omega)$ expresses the degree of possibility of $\omega$. Since one of the elements of $\Omega$ is the true value, it is assumed that $\pi(\omega)=1$ for at least one element $\omega$. The possibility measure of an event $A, \Pi(A)$, is defined by

$$
\Pi(A)=\sup _{\varpi \in \Omega} \pi(\varpi),
$$

and the necessity measure of $A, N(A)$, by

$$
N(A)=1-\Pi(\bar{A}) .
$$

Uncertainty about the occurrence of an event $A$, then, is represented by the couple $[N(A), \Pi(A)]$, where the necessity and possibility measures can be given the interpretation of probability bounds, i.e. lower and upper probabilities, respectively.

\section{AN IMPRECISION IMPORTANCE MEASURE}

Consider the system level reliability or risk parameter $p$ and its distribution pair $H_{p}$ induced by the propagation of a distribution pair $H_{q}$ through a model $g$. Define the imprecision of a distribution pair $H$, denoted $\Delta(H)$, as the area between its lower and upper cumulative distributions, i.e.

$\Delta(H)=\int(\max H(x)-\min H(x)) d x$.
For example, in the case of a distribution pair $H=$ $[N, \Pi]$ induced by a triangular possibility distribution $\pi$ with support $S$, we have - by geometrical considerations and recalling that a possibility distribution has unit height - that the imprecision of the possibility distribution is $\Delta(H)=|S| / 2$. In the case of a probabilistic representation of uncertainty we have $\max H(x)=\min H(x)$ for all $x$, and hence $\Delta(H)=0$.

Now define $\Delta_{i}\left(H_{p}\right)$ as the imprecision of $H_{p}$ when the imprecision of the distribution on the parameter $q_{i}$ is removed. We may then define an imprecision removal importance measure (IRIM) as

$$
\operatorname{IRIM}_{i}=\Delta\left(H_{p}\right)-\Delta_{i}\left(H_{p}\right),
$$

which expresses the amount of system level imprecision removal that comes from removing imprecision at the component level. The relative imprecision removal effect can be studied in terms of the measure

$$
\operatorname{RIRIM}_{i}=\frac{\Delta\left(H_{p}\right)-\Delta_{i}\left(H_{p}\right)}{\Delta\left(H_{p}\right)},
$$

which expresses the fraction of imprecision associated with the distribution pair $H_{p}$ that is attributable to component $i$.

As described in Section 1, imprecision can be removed either to the extent that a probabilistic representation remains, or to the extent that no epistemic uncertainty remains. Removal of imprecision to the extent that a probabilistic representation remains means that uncertainty about $q_{i}$ is described using a (subjective) probability distribution $F_{q i}(x)=$ $P\left(q_{i} \leq x\right)$, as illustrated in Figure 1.

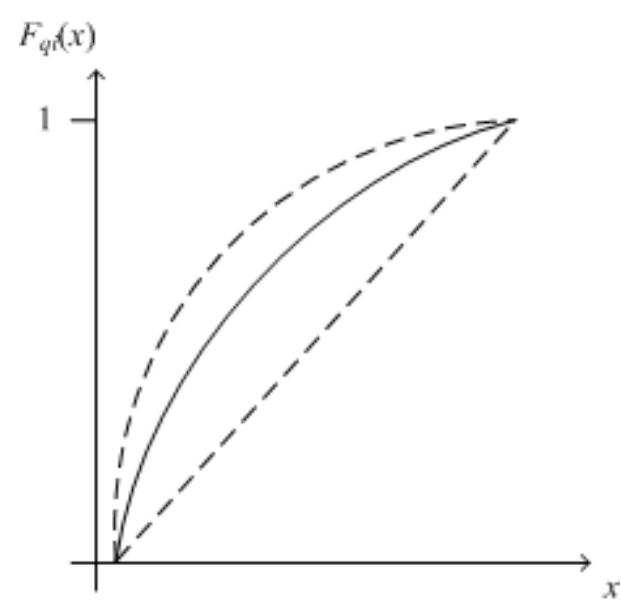

Figure 1. Removal of imprecision (imprecise probability sitribution - dashed lines) to the extent that a single-valued probabilistic representation remains (solid line).

Removal of imprecision to the extent that no epistemic uncertainty remains means that $q_{i}$ can be specified with certainty, and hence represented by $\delta_{q i}(x)$, where $\delta_{q i}(x)$ is the Dirac measure which equals 1 at $x=q_{i}$ and 0 otherwise, as illustrated in Figure 2. 


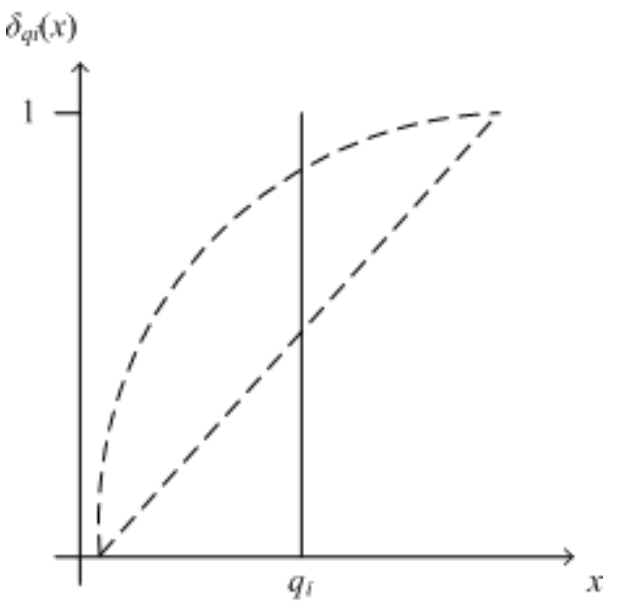

Figure 2. Removal of imprecision (imprecise probability sitribution - dashed lines) to the extent that a no epistemic uncertainty remains (solid line).

As described in Section 1, a substitute to imprecision removal to the extent that a probabilistic representation remains is the least and most specific probability distribution consistent with $H_{q}$. In the case of removal of imprecision to the extent that no imprecision remains, we are led to consider the suggested IIM as a function of $q_{i}$, denoted (R)IRIM ${ }_{i}^{\mathrm{II}}\left(q_{i}\right)$. In Section 5 we present a numerical example evaluating the latter type of measure.

\section{NUMERICAL EXAMPLE}

We consider a system $S$ consisting of three independent components, where component 1 and 2 are connected in a parallel configuration, which is again connected to component 3 in a series configuration. The reliability block diagram associated with system $S$ is shown in Figure 1.

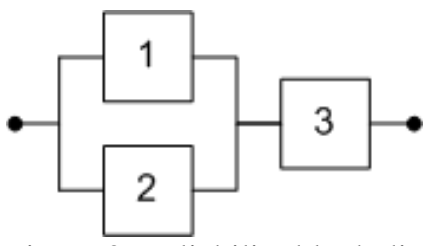

Figure 3. Reliability block diagram of system $\mathrm{S}$.

Component $i$ has availability $q_{i}, i=1,2,3$. The availability of the system, denoted $p$, is then

$$
p=\left(1-\left(1-q_{1}\right)\left(1-q_{2}\right)\right) q_{3} .
$$

The availability parameters $q=\left(q_{1}, q_{2}, q_{3}\right)$ are assumed to be unknown, the uncertainty being described using marginal necessity/possibility distribution pairs $H=\left(H_{1}, H_{2}, H_{3}\right)$, where $H_{i}(x)=\left[N\left(q_{i} \leq x\right)\right.$, $\left.\Pi\left(q_{i} \leq x\right)\right], i=1,2,3$.

We assume that the distribution pair $H_{1}$ is induced by a triangular possibility distribution $\pi_{1}$ with lower support 0.90 , mode 0.95 and upper support 0.99 ; that the distribution pair $\mathrm{H}_{2}$ is induced by a trapezoidal possibility distribution $\pi_{2}$ with lower support 0.92 , lower mode 0.94 , upper mode 0.96 and upper support 0.98; and that the distribution pair $\mathrm{H}_{3}$ is induced by a uniform possibility distribution $\pi_{3}$ with lower support 0.85 and upper support 0.95 . The component availability distributions and the resulting system availability distribution are illustrated in Figure 4.

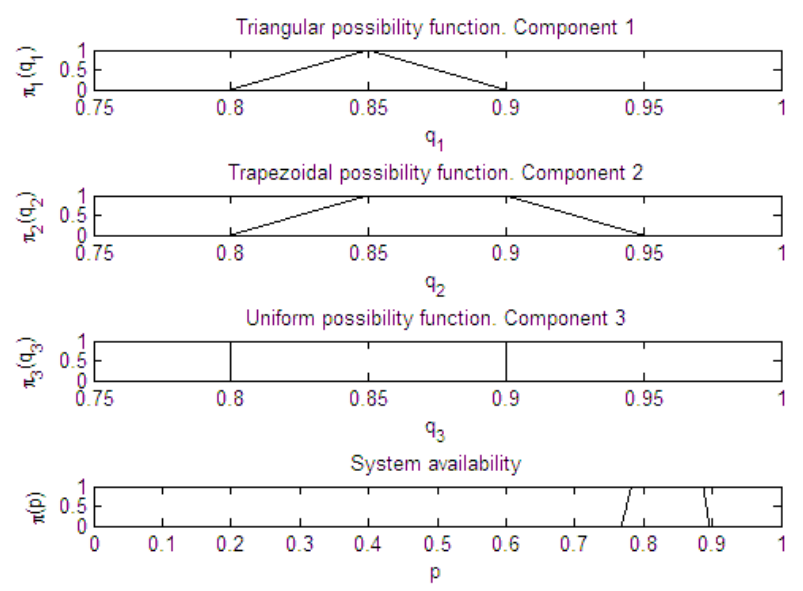

Figure 4. Input distribution functions on component availabilities and resulting system availability.

Let $s_{1}$ and $s_{2}\left(c_{1}\right.$ and $\left.c_{2}\right)$ denote the lower and upper support (core) limit of a possibility distribution, respectively. For a trapezoidal distribution we have $s_{1}$ $<c_{1}<c_{2}<s_{2}$, for a triangular distribution $s_{1}<c_{1}=c_{2}$ $<s_{2}$, and for a uniform distribution $s_{1}=c_{1}<c_{2}=s_{2}$. For these distribution classes we then have that the imprecision equals

$\Delta(H)=\frac{s_{2}-s_{1}+c_{2}-c_{1}}{2}$.

Table 1 lists the component and system availability distribution parameters as well as the associated imprecision index.

Table 1. Component and system availability distribution parameters and imprecision index for system $S$.

\begin{tabular}{llllll}
\hline$i$ & $s_{1}$ & $c_{1}$ & $c_{2}$ & $s_{2}$ & $\Delta\left(H_{i}\right)$ \\
\hline 1 & 0.80 & 0.85 & 0.85 & 0.90 & 0.05 \\
2 & 0.80 & 0.85 & 0.90 & 0.95 & 0.10 \\
3 & 0.80 & 0.80 & 0.90 & 0.90 & 0.10 \\
\hline System & 0.77 & 0.78 & 0.89 & 0.90 & 0.12 \\
\hline
\end{tabular}

Figure 5 shows the type II RIRIM as a function of $q_{i}$ for all the three components in system $S$. 


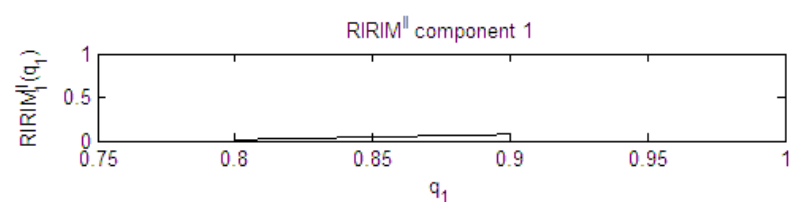

RIRIM" component 2

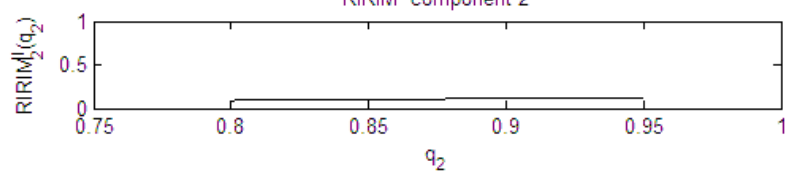

RIRIM" component 3

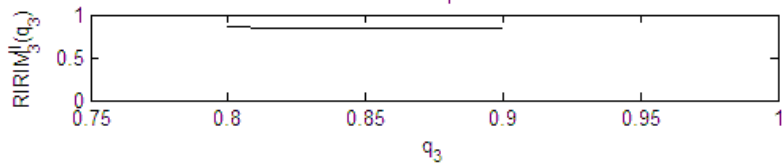

Figure 5. Type II RIRIM for each component of system S.

The (R)IRIM for each component is evaluated as a function of $\mathrm{q}_{\mathrm{i}}$ on the support of the associated distribution. Fixing the value of the availability of components 1 and 2 has the least imprecision removal effect on the system availability distribution. Depending on which values $q_{1}$ and $q_{2}$ are fixed at, the imprecision of the system availability distribution is reduced by a percentage between 1.29 and 7.54 for component 1 and between 9.05 and 12.6 for component 2. The greatest imprecision reduction can be obtained by fixing the value of the availability of component 3. By fixing $q_{3}$, the imprecision related to the system availability can be reduced by between 83.5 and 85.3 per cent. Table 2 summarises the value ranges of the (R)IRIM.

Table 2. Type II (R)IRIM value ranges.

\begin{tabular}{lll}
\hline$i$ & $\mathrm{IRIM}_{i}^{\mathrm{II}}$ & $\mathrm{RIRIM}_{i}^{\mathrm{II}}$ \\
\hline 1 & {$[0.0015,0.0087]$} & {$[1.29 \%, 7.54 \%]$} \\
2 & {$[0.0105,0.0146]$} & {$[9.05 \%, 12.6 \%]$} \\
3 & {$[0.0969,0.0990]$} & {$[83.5 \%, 85.3 \%]$} \\
\hline
\end{tabular}

\section{DISCUSSION AND FURTHER WORK}

In the present paper we have suggested an importance measure that can be used to evaluate the effect on system level parameter imprecision of removing component level parameter imprecision. Hence the suggested measure is defined analogously with the classical improvement potential IM which describes the effect of removing the unreliability of a component, and analogously with a number of UIMs that describe the effect of removing uncertainty about component performance.

Two extents of imprecision removal are considered: reduction to a probabilistic representation (type I) and removal of epistemic uncertainty (type II), the latter a special case of the former. Focus is put on the type II measure, and in a numerical example we study both the absolute and relative versions of the measure.
The relative version of the measure expresses the fraction of the initial amount of imprecision on the system level parameter that is attributable to each component. In a ranking setting this format is perhaps easier to comprehend than the underlying absolute numbers; however, the fractions need to be seen in relation to the initial amount of imprecision on the system level parameter.

Table 1 shows that component 2 and 3 have the same imprecision index when looked at in isolation, yet for the simple system example presented in Section 5 the conclusion is clearly that component 3 has the greatest imprecision importance. Looking at the structure function of system $\mathrm{S}$ it is clear that changes to the availability of component 3 will have the greatest direct effect on the system availability. In relation to system $\mathrm{S}$ it thus seems that the physical structure of the system is more important in terms of imprecision than is the shape of the individual distributions. For more complex systems and for other input distributions the conclusion may be less clear.

Further work in relation to the suggested measure is intended directed towards the implementation of the type II measure on more complex systems. Further work is also intended towards the development and implementation of the type I measure. Moreover, possibility theory provides a relatively simple and hence convenient uncertainty representation to use for the implementation of the suggested measures; however, other representations should also be considered in terms of application.

Finally, IIMs may be seen simply as a (natural) technical extension of UIMs when the uncertainty representation is no longer single-valued probability but instead some alternative representation with the interpretation of lower and upper probabilities. More interesting and potentially more decisiveis the role of IIMs in highlighting the relevance - for decisionmaking - of making the imprecision concept a central object of study in risk and reliability analysis. There is currently much interest and debate concerning the use of alternative representations of uncertainty in this setting, and sensibly defined and decision-relevant IIMs would be a relevant argument in this debate.

\section{ACKNOWLEDGEMENTS}

The work of E. Zio and P. Baraldi has been partially funded by the "Foundation pour une Culture de Securite' Industrielle" of Toulouse, France, under the research contract AO2009-04.

Terje: RAMONA??? 


\section{REFERENCES}

Apostolakis, G.E. 1990. The concept of probability in safety assessments of technological systems. Science 250(4986): 1359-1364.

Aven, T. \& Jensen, U. 1999. Stochastic Models in Reliability. New York: Springer.

Aven, T. \& Nøkland, T.E. 2010 On the use of uncertainty importance measures in reliability and risk analysis. Reliability Engineering and System Safety 95(2): 127-133.

Berger, J.O. 1984 The robust Bayesian viewpoint. In J. B. Kadane, JB (ed.), Robustness of Bayesian Analyses: 63-144. Amsterdam: Elsevier Science.

Borgonovo, E. 2006. Measuring uncertainty importance: Investigation and comparison of alternative approaches. Risk Analysis 26(5): 1349-1361.

Dempster, A.P. (1967) Upper and lower probabilities induced by a multivalued mapping. The Annals of Mathematical Statistics 38: 325-339.

Dubois, D. 2006. Possibility theory and statistical reasoning. Computational Statistics \& Data Analysis 51: 47-69.

Cheok, M.C., Parry, G.W. and Sherry, R.R. 1998. Use of importance measures in risk-informed regulatory applications. Reliability Engineering and System Safety 60: 213-226.

Dubois, D. \& Prade, H. 1988. Possibility Theory - An Approach to Computerized Processing of Uncertainty. New York: Plenum Press.

Ferson, S., Kreinovich, V., Ginzburg, L., Myers, D.S. \& Sentz, K. 2003. Constructing probability boxes and DempsterShafer structures. Technical Report SAND2002-4015, Sandia National Laboratories.

Helton, J C \& Burmaster, D E (1996) Guest editorial: treatment of aleatory and epistemic uncertainty in performance assessments for complex systems. Reliability Engineering and System Safety 54: 91-94.

Iman, R.L. 1987. A matrix-based approach to uncertainty and sensitivity analysis for fault trees. Risk Analysis 7(1): 21-33.

Kaplan, S. \& Garrick, B.J. 1981. On the quantitative definition of risk. Risk Analysis 1(1): 11-27.

Nøkland, T.E. \& Aven, T. unpubl. On selection of importance measures in risk and reliability analysis.

Rausand, M. \& Høyland, A. 2004. System Reliability Theory: Models, Statistical Methods, and Applications. 2nd ed. Hoboken, N.J.: Wiley-Interscience.

Shafer, G. 1976. A Mathematical Theory of Evidence. Princeton University Press.

Walley, P. 1991. Statistical Reasoning with Imprecise Probabilities. London: Chapman and Hall.

Weichselberger, K. 2000. The theory of interval probability as a unifying concept for uncertainty. International Journal of Approximate Reasoning 24: 149-170.

Zio, E. 2009. Computational Methods for Reliability and Risk Analysis. Hackensack, N.J.: World Scientific. 